\title{
ESCARABAJOS ACUÁTICOS (COLEOPTERA: ADEPHAGA, POLYPHAGA) DE LA RESERVA FLORÍSTICA MANEJADA MONTE BARRANCA, SANTIAGO DE CUBA, CUBA
}

\author{
${ }^{1}$ Albert Deler-Hernández y ${ }^{2}$ Franklyn Cala-Riquelme \\ Centro Oriental de Ecosistemas y Biodiversidad (BIOECO). Enramadas \# 601, Esq. Barnada, Santiago de \\ Cuba, 90100, Cuba. ${ }^{1}$ adeler1982@yahoo.com; ${ }^{2}$ franklyn@bioeco.ciges.inf.cu
}

RESUMEN

Se realizó un estudio de la fauna de escarabajos acuáticos de la Reserva Florística Manejada Monte Barranca. Se reportaron un total de 264 ejemplares, distribuidos en 5 familias, 17 especies y una subespecie.

Palabras Clave: Coleoptera, Adephaga, Polyphaga, Cuba.

\section{ABSTRACT}

A study of coleoptera aquatic fauna in the protected area Monte Barranca was conducted. A total of 264 specimens, 5 families, 17 species and 1 subspecie are recorded for this protected area.

Key words: Coleoptera, Adephaga, Polyphaga, Cuba.

\section{INTRODUCCIÓN}

El orden Coleoptera no solo tiene un alto número de especies, también posee una gran diversidad de formas, tamaños y estrategias ecológicas. Aunque la mayoría de los escarabajos son terrestres, por lo menos 10,000 especies son consideradas acuáticas en una o más de sus fases de desarrollo (Balke et al., 2004). De los cuatro subórdenes en los que está dividido el Orden, tres tienen representantes acuáticos, aunque el mayor número de especies pertenece a los subórdenes Adephaga y Polyphaga (Whiteman y Sites, 2003).

Los adéfagos acuáticos o Hydradephaga como también son llamados están compuestos por ocho familias, de las cuales cuatro, Dytiscidae (48), Gyrinidae (6), Haliplidae (7), Noteridae (10) están presentes en Cuba (Nilsson, 2001; Nilsson, 2003; Nilsson y Vondel, 2005; Vondel y Spangler, 2008).

Polyphaga es el grupo más diverso del orden Coleoptera, con alrededor del $90 \%$ de todas las especies de escarabajos (Lawrence y Britton, 1991). En Cuba están presentes siete familias acuáticas: Hydrophilidae (33), Hydrochydae (4), Hydraenidae (4), Dryopidae (1), Elmidae (4), Psephenidae (1) y Lutrochidae (1) (Peck, 2005; Shepard y Megna, 2006).

La fauna de coleópteros acuáticos de Cuba necesita de inventarios actualizados, así como una caracterización desde el punto de vista faunístico que permita en primer lugar un conocimiento profundo de la especie como objeto de estudio, la extrapolación de los datos obtenidos a otras áreas similares, y la conservación de estas especies y otros grupos de animales y vegetales asociados a ellas.

El presente estudio está dirigido a realizar una lista taxonómica de la fauna de escarabajos acuáticos de la Reserva Florística Manejada Monte Barranca, además de aportar datos sobre su distribución y ecología.

\section{MATERIALES Y MÉTODOS}

La Reserva Florística Manejada Monte Barranca es un relicto de bosque Semideciduo Mesófilo, con una extensión superficial de 301 ha y una altura de 203 m.s.n.m., a $39 \mathrm{Km}$ al noroeste de 
la ciudad de Palma Soriano (Santiago de Cuba). A pesar de su poca extensión, posee una gran riqueza de flora y fauna, dado principalmente por el intercambio de biota entre los macizos montañosos SaguaBaracoa y Sierra Maestra.

Los muestreos se realizaron en diciembre de 2007 y diciembre de 2008. En el área de estudio, fueron seleccionados todos los ambientes acuáticos y caracterizados según los siguientes datos (Tabla 1):

VEGE: Vegetación. No(1), presencia (2).

FOND: Tipo de fondo. Se determinaron 2 categorías: fangoso (1) y fangoso-arenoso (2).

GINS: Grado de insolación. Los ambientes acuáticos con más del $60 \%$ del espejo de agua sombreado por el dosel de los árboles fueron considerados con una gradación baja (1) y aquellos que tenían menos del $60 \%$ con una gradación alta (2).

DETR: Detritus. No(1), presencia (2).

TURB: Turbidez. Este parámetro fue evaluado considerando la transparencia del agua: aguas claras (1) se corresponden con aquellos hábitats donde era posible observar con facilidad el fondo, y aguas turbias (2) aquellos donde no se observa el fondo.

SALI: Salinidad. Se definieron dos tipos: aguas dulces (1) y aguas salobres (2).

Para la colecta de los especímenes se empleó una red de mano triangular de $30 \mathrm{~cm}$ en cada lado, $40 \mathrm{~cm}$ de fondo, con un ancho de la malla inferior a $0,01 \mathrm{~mm}$ y mango de $1 \mathrm{~m}$. Todos los ejemplares colectados fueron conservados en alcohol al $75 \%$, y etiquetados para su posterior identificación en el Laboratorio de Zoología del Departamento de Biología de la Universidad de Oriente.

\title{
RESULTADOS
}

\section{LISTA ANOTADA DE LAS ESPECIES DE ESCARABAJOS ACUÁTICOS DE LA RESERVA FLORÍSTICA MANEJADA MONTE BARRANCA, SANTIAGO DE CUBA.}

Se colectaron 264 ejemplares, distribuidos en 5 familias, 17 especies y una subespecie. El mayor número de ejemplares corresponde a la familia Dytiscidae con 110 especímenes, luego Hydrophilidae con 108, Hydraenidae con 41, Hydrochidae con 4 y Haliplidae con 1 (Tabla 2). (* especie endémica).

\author{
Suborden Adephaga \\ Familia Dytiscidae Leach \\ Subfamilia Copelatinae Branden \\ Copelatus coelatipennis angustatus Chevrolat 1863
}

Diagnosis. Élitros con 10 estrías longitudinales y una estría posterolateral submarginal, protibia del macho sin emarginación ventrobasal.

C. c. angustatus es conocida para el sur de la Florida (Epler, 1996). Ampliamente distribuida en Cuba (Megna y Alarie, datos sin publicar).

Subfamilia Colymbetinae Erichson

Rhantus calidus (Fabricius, 1792)

Diagnosis. Ojos emarginados por la base de las antenas, proceso prosternal y prosterno medialmente convexo, pro- y mesotarsos con cinco segmentos, uñas metatarsales desiguales. 
Esta especie tiene un amplio rango de distribucción en América, abarcando la región Neártica y Neotropical (Balke et al., 2002). En Cuba ha sido reportada en todas las regiones del país, incluyendo la Isla de la Juventud.

$R$. calidus es el único representante del género en Cuba. Sus especímenes han sido colectados en ambientes lénticos y lóticos de aguas claras y turbias.

Subfamilia Laccophilinae Gistel

Laccophilus proximus Say, 1823

Diagnosis. Élitros irrorados, pronoto testáceo sin mancha anterior oscura, metacoxa del macho con órgano estridular.

L. proximus está distribuida desde el sur de Canadá hasta la América Central y las Antillas (Zimmerman, 1970; Spangler, 1981). Es una de las especies más abundantes de la familia Dytiscidae y es considerada como una de las de más amplia distribución en Cuba (Peck, 2005). Los adultos de L. proximus están frecuentemente presentes en todos los hábitats de agua dulce descritos (Megna y Deler-Hernández, En prensa).

\section{Subfamilia Dytiscinae Leach \\ Hydaticus bimarginatus (Say, 1830)}

Diagnosis. Élitros con banda amarilla lateral, sin bandas sublaterales; metatibia con más de 10 setas (vista dorsal).

Especie presente en Estados Unidos y las Antillas. En Cuba ha sido reportada para el Centro y Oriente de país. Aunque todas las especies de $H$. bimarginatus han sido colectadas en hábitats lénticos (temporales y permanentes), en este estudio los ejemplares fueron colectados en remansos de hábitats lóticos de aguas turbias, con fondo fangoso y abundante acumulación de materia orgánica.

\section{Thermonectus circumscriptus (Latreille, 1809)}

Diagnosis. Pronoto amarillo con el margen anterior y posterior con una banda negra.

Ampliamente distribuida en Cuba. Especie muy frecuente en ambientes lénticos permanentes y en remansos de hábitats lóticos (Megna et al., 2009).

Familia Haliplidae Aubé

Haliplus youngi Vondel y Spangler, 2008

Diagnosis. Tamaño superior a los $3.0 \mathrm{~mm}$. Margen anterior de los élitros con alrededor de 20 dientes.

En Cuba hay reportadas seis especies del género Haliplus Latreille, de las cuales dos son endémicas (Vondel y Spangler, 2008). Esta especie, determinada como H. tumidus Leconte (Spangler, 1981) fue reportada para Arroyo Jarahueca (Mayarí Arriba), por lo que en este estudio se amplía su rango de distribución. El espécimen fue colectado entre la vegetación de orilla, en ambiente lótico de aguas turbias.

Suborden Polyphaga Emery

Familia Hydrophilidae Latreille

Subfamilia Hydrophilinae Latreille 


\section{CLAVE PARA ADULTOS DE HYDROPHILIDAE ACUÁTICOS DE LA RESERVA FLORÍSTICA MANEJADA MONTE BARRANCA, SANTIAGO DE CUBA.}

1 Proceso meso- y metasternal forman una gran quilla continua, en forma de espina. .2

Proceso meso- y metasternal sin una gran quilla continua, en forma de espina..................5

2 Especies con tamaño mayor de $30.0 \mathrm{~mm}$ H. ensifer

Especies con tamaño menor de $20.0 \mathrm{~mm}$. .3

3 Carena prosternal con prolongación en forma de espina en el margen posterior. .H. casta Prosterno sin carena.

5 Labro oculto por el clípeo. H. larvalis Labro no oculto por el clípeo.

6 Tibias medias y posteriores con hileras de setas natatorias; escutelo mucho más largo que ancho; segundo segmento de los palpos maxilares normales Tibias medias y posteriores sin hileras de setas natatorias; escutelo casi tan largo como ancho; segundo segmento de los palpos maxilares inclinados hacia afuera.....

7 Cabeza testácea; emarginación del $5^{\text {to }}$ esternito abdominal en forma de herradura. B. quadridens Cabeza negra; emarginación del $5^{\text {to }}$ esternito abdominal con dos dientes. B. undatus

8 Ápice de los parámeros curvados hacia afuera E. pseudochraceus Ápice de los parámeros ligeramente estrechos. E. aequalis

\section{Enochrus aequalis (Sharp, 1882)}

Diagnosis. Prosteno no carinado; cresta mesosternal larga y ancha.

Especie presente en América Central y las Antillas. En Cuba es de amplia distribución. Colectada sólo en hábitats lóticos.

\section{Enochrus pseudochraceus Gundersen, 1977}

Diagnosis. Prosterno no carinado; cresta mesosternal baja, con el ápice recurvado formando un pequeño diente en la parte posterior. Parámeros con ápices curvados hacia afuera y ápice del lóbulo medio ancho y lanceolado.

Especie presente en América Central y las Antillas. E. pseudochraceus es una de las más abundantes y la más distribuida del género en Cuba. Aunque ha sido colectada en todos los hábitats acuáticos descritos; la especie tiene cierta preferencia por los ambientes lóticos, donde es muy frecuente colectarla en las márgenes de ríos y arroyos, entre las rocas y la vegetación de orilla.

\section{Helobata larvalis (Horn, 1873)}

Diagnosis. Tamaño menor a los $6.5 \mathrm{~mm}$. Clípeo grande, con borde anterior redondeado o recto que oculta al labro.

Distribuida en toda América. En Cuba sólo ha sido reportada en el Oriente del país. Su presencia en los ambientes acuáticos es muy rara, ha sido colectada en hábitat lóticos de aguas turbias, con abundante detritus y presencia de vegetación de orilla.

\section{Hydrobiomorpha casta (Say, 1835)}

Diagnosis. Forma elíptica, carena prosternal con punta posterior larga, área glabra subtriangular o ausente.

H. casta se extiende desde el sur de Estados Unidos hasta América Central y el Caribe. Única especie del género en Cuba. Sus especímenes no son muy abundantes en los hábitats acuáticos cubanos, ha sido reportada sólo en hábitats lóticos. 
Hydrophilus ensifer Oliver, 1792

Diagnosis. Tamaño de 30.0 a $33.0 \mathrm{~mm}$. Área glabra del $2^{\text {do }}$ urosternito abdominal visible, pequeño y en forma triangular.

H. ensifer está presente en América Central, el Caribe y América del Sur. Especie muy rara. Sus ejemplares han sido colectados en remansos de hábitats lóticos.

Tropisternus collaris (Fabricius, 1775)

Diagnosis. Élitros con varias bandas longitudinales verdosas metálicas en toda su longitud. Surco prosternal abierto anteriormente.

T. collaris es conocida para las Antillas y América del Sur. Ampliamente distribuida en Cuba.

Tropisternus lateralis (Fabricius, 1775)

Diagnosis. Borde lateral de los élitros, pronoto y cabeza testáceo.

Especie de amplia distribución en América considerada como una de las primeras especies de escarabajos acuáticos, capaz de establecerse en todos los hábitats acuáticos disponibles (Spangler, 1981).

Berosus undatus (Fabricius, 1792)

Diagnosis. Tamaño de 5.0 a $7.0 \mathrm{~mm}$. Ápice elitral con una proyección en forma de espina. Cabeza negra. Escotadura del $5^{\text {to }}$ esternito abdominal con dos dientes en la parte media; carena entre las coxas del $1^{\text {er }}$ esternito abdominal. Genitalia del macho con lóbulo medio fuertemente arqueado en vista lateral.

Especie reportada en México y las Antillas. En Cuba ha sido reportada para la región Oriental. Colectada en hábitats lénticos y lóticos (remansos).

\section{Berosus quadridens Chevrolat, 1863}

Diagnosis. Tamaño de 5.0 a $7.5 \mathrm{~mm}$. Ápice elitral con una proyección en forma de espina. Cabeza testácea. Escotadura del $5^{\text {to }}$ esternito abdominal con emarginación profunda en forma de herradura. Genitalia del macho con parámeros largos y unidos en el ápice.

Especie reportada en América Central y las Antillas. Descrita por Chevrolat (1863) para Cuba, a partir de una hembra de la colección de D. F. Poey. Colectada en ambos tipos de hábitats, siempre asociada con B. undatus.

Familia Hydraenidae Mulsant

Subfamilia Hydraenine Mulsant

Hydraena perkinsi Spangler, 1980 *

Diagnosis. Espacio estrecho entre las placas metasternales.

De las dos especies del género presentes en Cuba, es $H$. perkinsi la más abundante y la de más amplia distribución. Ha sido encontrada en una gran variedad de hábitats (lénticos y lóticos). En el área de estudio ha sido colectada en ambientes lóticos ecológicamente muy diferentes.

Subfamilia Ochthebiinae Thomson

Gymnochthebius fossatus (LeConte, 1855)

Diagnosis. Pieza principal del edeago bifurcada en su ápice.

Especie que se extiende desde el sur de Estados Unidos hasta Argentina (Perkins, 1980; Delgado et al., 1995). En Cuba ha sido colectada en una amplia variedad de hábitats (lénticos y lóticos). En el área de estudio se colectó en remansos de aguas turbias, con abundante materia orgánica y fondo fangoso.

\section{Familia Hydrochidae Thomson}

Hydrochus sp.

Especie colectada de forma directa sobre troncos en descomposición. 
Tabla 1. Características ecológicas de las estaciones muestreadas en la Reserva Florística Manejada Monte Barranca.

\begin{tabular}{|c|c|c|c|c|c|c|c|c|}
\hline \multicolumn{2}{|c|}{ Estación } & Fecha & $\begin{array}{l}\mathrm{V} \\
\mathrm{E} \\
\mathrm{G} \\
\mathrm{E}\end{array}$ & $\begin{array}{l}\mathrm{F} \\
\mathrm{O} \\
\mathrm{N} \\
\mathrm{D}\end{array}$ & $\begin{array}{l}\mathrm{G} \\
\mathrm{I} \\
\mathrm{N} \\
\mathrm{S}\end{array}$ & $\begin{array}{l}\mathrm{T} \\
\mathrm{U} \\
\mathrm{R} \\
\mathrm{B}\end{array}$ & $\begin{array}{l}\mathrm{S} \\
\mathrm{A} \\
\mathrm{L} \\
\mathrm{I}\end{array}$ & $\begin{array}{l}\mathrm{D} \\
\mathrm{E} \\
\mathrm{T} \\
\mathrm{R}\end{array}$ \\
\hline \multirow{2}{*}{ E-1 } & \multirow{2}{*}{ Arroyo: remanso } & 04-XII-2007 & 2 & 1 & 2 & 2 & 1 & 2 \\
\hline & & 21-XII-2008 & 2 & 1 & 2 & 2 & 1 & 2 \\
\hline \multirow{2}{*}{ E-2 } & \multirow{2}{*}{ Arroyo: remanso } & 04-XII-2007 & 2 & 2 & 1 & 1 & 1 & 1 \\
\hline & & 22-XII-2008 & 2 & 2 & 1 & 1 & 1 & 1 \\
\hline \multirow{2}{*}{ E-3 } & \multirow{2}{*}{ Presa Monte Barranca } & 05-XII-2007 & 2 & 2 & 2 & 2 & 1 & 1 \\
\hline & & 22-XII-2008 & 2 & 2 & 2 & 2 & 1 & 1 \\
\hline
\end{tabular}

Tabla 2. Riqueza y abundancia de especies por estaciones de colectas en la Reserva Florística Manejada Monte Barranca.

\begin{tabular}{|c|c|c|c|c|}
\hline \multirow[b]{2}{*}{ ESPECIES } & \multicolumn{4}{|c|}{ Estaciones de Colecta } \\
\hline & E-1 & E-2 & E-3 & Total \\
\hline \multicolumn{5}{|l|}{ Dytiscidae } \\
\hline 1. C. c.angustatus Chevrolat & 7 & - & 3 & 10 \\
\hline 2. $R$. calidus (Fabricius) & 5 & 1 & 6 & 12 \\
\hline 3. L. proximus Say & - & - & 60 & 60 \\
\hline 4. H. bimarginatus (Say) & 2 & - & - & 2 \\
\hline 5. T. circumscriptus (Latreille) & 26 & - & - & 26 \\
\hline \multicolumn{5}{|l|}{ Haliplidae } \\
\hline 6. Haliplus youngi & - & - & 1 & 1 \\
\hline \multicolumn{5}{|l|}{ Hydrophilidae } \\
\hline 7. E. aequalis (Sharp) & 10 & - & 20 & 30 \\
\hline 8. E. pseudochraceus Gundersen & 16 & - & 4 & 20 \\
\hline 9. H. larvalis (Horn) & 4 & - & - & 4 \\
\hline 10. H. casta (Say) & - & - & 2 & 2 \\
\hline 11. H. ensifer Oliver & - & - & 3 & 3 \\
\hline 12. T. collaris (Fabricius) & - & - & 9 & 9 \\
\hline 13. T. lateralis (Fabricius) & - & - & 29 & 29 \\
\hline 14. B. undatus (Fabricius) & - & - & 8 & 8 \\
\hline 15. B. quadridens Chevrolat & - & - & 3 & 3 \\
\hline \multicolumn{5}{|l|}{ Hydraenidae } \\
\hline 16. H. perkinsi Spangler* & 15 & 8 & 6 & 29 \\
\hline 17. G. fossatus (LeConte) & 12 & - & - & 12 \\
\hline \multicolumn{5}{|l|}{ Hydrochidae } \\
\hline 18. Hydrochus $s p$ & 3 & - & 1 & 4 \\
\hline Totales & 100 & 9 & 155 & 264 \\
\hline
\end{tabular}




\section{AGRADECIMIENTOS}

A todos los técnicos y trabajadores de la Reserva Florística Manejada Monte Barranca y al Departamento de Conservación de la Empresa Flora y Fauna, Santiago de Cuba. Al Lic. Bruno Téllez (Universidad de Oriente) por su ayuda en el trabajo de campo y al Lic. Yoandri Suárez Megna (Universidad de Oriente) por su ayuda en la identificación de los Dytiscidae.

\section{LITERATURA CITADA}

Balke, M., R. E. Roughley, W. Sondermann y P. J. Spangler. 2002. Diving beetles of the genus Rhantus in Costa Rica: Taxonomy and Biogeography, with notes on South American species (Coleoptera: Dytiscidae). Study on Neotropical Fauna and Environment 37: 263-271.

Balke, M., M. A. Jäch y L. Hendrich. 2004. Insecta: Coleoptera. In: Catherine Mary Yule \& Yong Hoi Sen (eds.). Freshwater Invertebrates of the Malaysian Region. Academy of Sciences Malaysia, pp.

Delgado, J. A, F. Collantes y A. G. Soler. 1995. Gymnochthebius fossatus (Leconte, 1885) cita interesante para Nicaragua (Coleoptera: Hydraenidae). Revista Nicaragüense de Entomología 33: $13-16$.

Epler, J.H. 1996. Identification manual for the water beetles of Florida (Coleoptera: Dryopidae, Dytiscidae, Elmidae, Gyrinidae, Haliplidae, Hydraenidae, Hydrophilidae, Noteridae, Psephenidae, Ptilodactylidae, Scirtidae). Florida Department of Environmental Protection. Tallahassee, iv +253 unnumbered pp.

Lawrence, J. F. y E. B. Britton. 1991. Coleoptera (Beetles). In: CSIRO The Insects of Australia Vol. 2. Melbourne University Press. pp 542-1137.

Megna, Y. S. y A. Deler-Hernández. (In press). The Dytiscidae (Coleoptera) of Cuba I. The subfamily Laccophilinae. Zootaxa.

Megna, Y. S., Y. Alarie y A. Deler-Hernández. 2009. First West Indies records of Thermonectus succinctus (Aubé, 1838) (Coleoptera: Dytiscidae), with notes on other Cuban species. Koleopterologische Rundschau 79: 5-17.

Nilsson, A. N. 2001. World Catalogue of Insects. Vol. 3. Dytiscidae (Coleoptera). Apollo Books, Stenstrup, Denmark. 395 pp.

Nilsson, A. N. 2003. World catalogue of Noteridae. Internet version 2003-12-05. www.bmg.umu.se/biginst/andersn/wen/wen_index.htm.

Nilsson, A. N. y B. J. van Vondel. 2005. Amphizoidae, Aspidytidae, Haliplidae, Noteridae, and Paelobiidae (Coleoptera, Adephaga). In: World Catalogue of Insects 7: 1-171. Apollo Books. Stenstrup, Denmark.

Peck, S. B. 2005. A checklist of the beetles of Cuba with data on distribution and bionomics (Insecta: Coleoptera). Arthropods of Florida and neighboring land areas 18: 1-241.

Perkins, P. D. 1980. Aquatic beetles of the family Hydraenidae in the Westen Hemisphere: Classification, Biogeography, and Inferred Phylogeny (Insecta: Coleoptera). Quaestiones Entomolgicae 16: 3-554. 
Shepard, W. D y Y. S. Megna. 2006. Los byrroideos acuáticos (Coleoptera: Byrroidea) de Cuba. Cocuyo 16:32-35.

Short, A. E. Z. 2005. A review of the subtribe Acidocerina of Central America with special reference to Costa Rica (Coleoptera: Hydrophilidae). Koleopterologische Rundschau 75: 191-226.

Spangler P. J. 1981. Suplement to the aquatic and semiaquatic Coleoptera of Cuba collected by the Biospeleological Expeditions to Cuba by the Academies of Science of Cuba and Romania. Résultats des Expéditions Biospéologiques Cubano-Roumaine a Cuba 3: 145-171.

Vondel B. J. van y P.J. Spangler. 2008 Revision of the Haliplidae of the Neotropical Region including Mexico (Coleoptera: Haliplidae). Koleopterologische Rundschau 78: 69-194.

Whiteman, N.K. Y Sites, R. 2003. Lentic beetles of the Missouri prairie region: habitat and regional associations, with keys to the Hydradephaga. Transactions of the American Entomological Society 129: 185243.

Zimmerman, J.R. 1970. A taxonomic revision of the aquatic beetles genus Laccophilus (Dytiscidae) of the North America. Memoires of the American Entomological Society, 26: 1-275. 